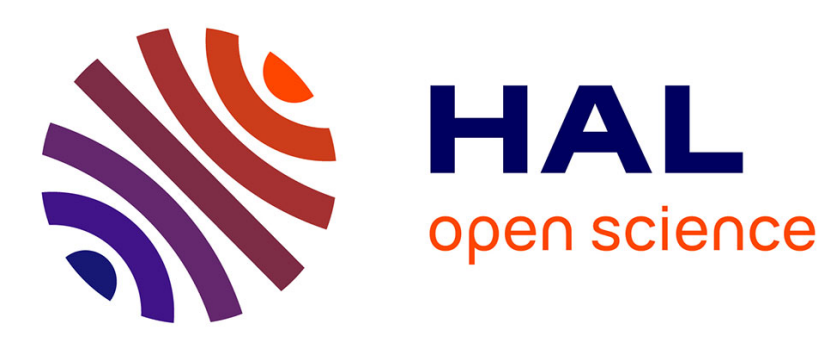

\title{
2D Manipulation of Nanoobjects by Perpendicular Electric Fields: Implications for Nanofabrication
}

Stefano Curiotto, F. Cheynis, Pierre Müller, Frédéric Leroy

\section{To cite this version:}

Stefano Curiotto, F. Cheynis, Pierre Müller, Frédéric Leroy. 2D Manipulation of Nanoobjects by Perpendicular Electric Fields: Implications for Nanofabrication. ACS Applied Nano Materials, 2020, 3 (2), pp.1118-1122. 10.1021/acsanm.9b02517 . hal-02499335

\section{HAL Id: hal-02499335 https://hal-amu.archives-ouvertes.fr/hal-02499335}

Submitted on 5 Mar 2020

HAL is a multi-disciplinary open access archive for the deposit and dissemination of scientific research documents, whether they are published or not. The documents may come from teaching and research institutions in France or abroad, or from public or private research centers.
L'archive ouverte pluridisciplinaire HAL, est destinée au dépôt et à la diffusion de documents scientifiques de niveau recherche, publiés ou non, émanant des établissements d'enseignement et de recherche français ou étrangers, des laboratoires publics ou privés. 


\title{
2D Manipulation of Nano-Objects by Perpendicular Electric Fields: Implications for Nanofabrication
}

\author{
Stefano Curiotto, ${ }^{*}$ Fabien Cheynis, ${ }^{*}$ Pierre Müller, ${ }^{*}$ and Frédéric Leroy* \\ Aix Marseille Univ, CNRS, CINAM, Marseille, France \\ E-mail: curiotto@cinam.univ-mrs.fr; cheynis@cinam.univ-mrs.fr; muller@cinam.univ-mrs.fr; \\ leroy@cinam.univ-mrs.fr
}

This manuscript is a pre-print, the final version has been published in ACS Applied Nano Materials, 3 (2020) 1118-1122

Publication Date:February 4, 2020

https://doi.org/10.1021/acsanm.9b02517

\section{Abstract}

The capability to control the motion of nanoobjects on a surface would open perspectives in nanofabrication. Here, we show the proof of concept that the displacement of a 2D nanostructure on a surface can be controlled by means of two perpendicular electric fields. With a specifically designed sample holder, we displace a $2 \mathrm{D}$ negative island on $\mathrm{Si}(001)$ along a close loop, in a low energy electron microscope. Our technique could be applied to other systems, to assemble nanomaterials.

Keywords: surfaces, low energy electron microscopy, silicon, electromigration, nano assembly

The development of techniques to fabricate networks of ordered nano-structures on surfaces, like nanowires ${ }^{1,2}$, nanotubes ${ }^{3}$, or nanoislands ${ }^{4,5}$ widens horizons in nanodevice engineering. A different approach in nanofabrication consists in positioning single nano-objects on a surface. For instance, top-down approaches are used to directly fabricate nanostructures at defined positions ${ }^{6}$.
Controlling the position of an already formed object by moving it on a surface is more delicate. To this aim, a challenge among different research groups was launched with the NanoCar race ${ }^{7,8}$. The ultimate way to move nano objects is the manipulation of single atoms, as performed by Eigler and Schweizer by scanning tunneling microscopy (STM) with Xe atoms ${ }^{9}$. This procedure is very precise and ideally suited for very small clusters. However, as it involves the displacement of each single atom by a STM tip, it is slow and complex to implement. Another way to move nano objects could pass through the exploitation of external fields. Electric fields are known to lead to mass displacement through the electromigration phenomenon. They can either exert a direct force on partially charged atoms, or they can induce an electric current and the momentum transfer between moving electrons and atoms induces preferential atomic diffusion in the electron direction ("electron wind" force $)^{10}$. Historically, electromigration has been considered a detrimental effect because, if not controlled, it can induce accumulation or depletion of matter at specific locations, with the formation of hillocks and voids ${ }^{11}$ that lead to short circuits in electronic devices. More recently, as suggested by Kevrekidis ${ }^{12}$, the idea that external fields can be a tool for surface nanopattern engineering started to spread. Our work is motivated by the 
perspective of controlling the position of single nano-objects on a surface. Here, we show the proof of concept that precise control of the $2 \mathrm{D}$ displacement of a nanostructure by means of electric fields can be carried out.

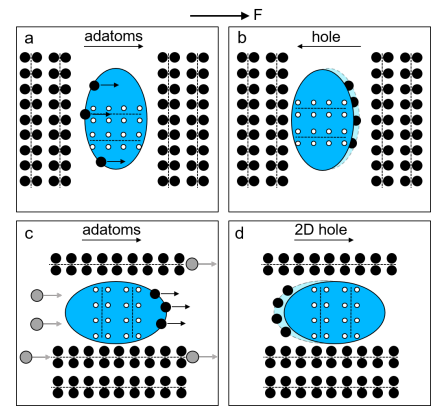

Figure 1: Schematics of the motion of 2D one-atom-deep holes (blue ellipses) in $\mathrm{Si}(001)$. Their shape is elliptical because of the anisotropy of the step edge stiffness. Dark circles represent $\mathrm{Si}$ atoms of the terrace outside (higher than) the hole. Small bright circles are atoms of the terrace inside the hole. Large grey circles are adatoms on the terrace outside the hole. In $\mathrm{a}$ and $\mathrm{b}$ the dimer rows outside the hole are perpendicular to the electric field that generates a force on the adatoms from left to right. Because of the diffusion anisotropy, the adatoms move fast inside the $2 \mathrm{D}$ hole, where the dimer rows are parallel to the force and they move slowly outside, perpendicularly to the dimer rows. They detach from the left edge of the hole and attach to the right edge. Macroscopically, the hole moves to the left. The light blue region in $b$ represents the position occupied by the hole before the motion, in a. In $\mathrm{c}$ and $\mathrm{d}$, the dimer rows outside the hole are parallel to the electric field. Adatoms move from left to right along the dimer rows, they attach to the left edge of the hole and detach from the right edge. In this case the $2 \mathrm{D}$ hole moves to the right, in the same direction of the electric field.

We have chosen the $\mathrm{Si}(001)$ surface and 2D negative islands (one-atom-deep holes) as model nanostructures. The effect of electromigration on this surface has been widely studied $^{13-19}$, and the relation between electric-field direction and the motion of $2 \mathrm{D}$ nanostructures has been recently described ${ }^{20}$. The $\mathrm{Si}(001)$ surface reconstructs by forming parallel dimer rows. The direction of these rows rotates by $90^{\circ}$ on successive terraces ${ }^{21}$. Because of this structural anisotropy, the diffusion of atoms adsorbed on the surface is faster along the dimer rows than perpendicularly to them ${ }^{22}$. On $\mathrm{Si}(001)$, an electric field facilitates adatom diffusion in the electric current direction ${ }^{23}$. When an external force $F$ affects the diffusion of adatoms, the velocity $v$ of a single adatom in the force direction can be written using the Nernst-Einstein relation: $v=\frac{F \cdot D_{a d}}{k_{B} T}$, where $D_{a d}$ is the adatom diffusion coefficient, $k_{B}$ is the Boltzmann constant and $T$ is the temperature. A flux of adatoms does not necessarily imply motion of nanostructures. 2D holes can move if the adatom fluxes inside and outside the hole are unbalanced, i.e. if there is an asymmetry between the inner and the outer part of the hole. If diffusion inside the hole were as fast as diffusion outside, the number of atoms arriving to an edge would equal the number of atoms leaving that edge and the hole would not move. Therefore the specific material surface plays an important role in the motion of the nanostructure. Actually, an edge often implies an asymmetry of some properties. For $\mathrm{Si}(001)$ the edge of a 2D hole marks a surface structure change, as the dimer rows inside and outside the 2D hole have different orientations. For other materials, the Ehrlich-Schwoebel barrier ${ }^{24,25}$, differences in adatom attachment/detachment at the edge could generate asymmetries between the edge sides and thus enable the motion of a nanostructure under an electric field.

Figure 1 shows a schematics of the motion of two different holes under an external force. The case of a force parallel to the direction of the dimer rows inside the $2 \mathrm{D}$ hole is described in figures $1 \mathrm{a}$ and $1 \mathrm{~b}$. Outside the hole the force is perpendicular to the fast-diffusion direction (the direction of the dimer rows), and thus its effect is weaker. Inside the hole, the force is parallel to the fast diffusion direction and adatoms drift preferentially in the force direction. Adatoms detach from the left edge, diffuse towards the right and attach to the right edge, thus the 2D hole moves to the left, opposite to the force. For the 2D hole shown in figures $1 \mathrm{c}$ 
and 1d, the force on the adatoms has only a weak effect inside the hole, where it is perpendicular to the fast diffusion direction. Outside the $2 \mathrm{D}$ hole, the force makes the adatom drift from left to right. Therefore adatoms attach to the left edge of the hole, while they are detached from the right edge to move towards the right. The hole moves from left to right, in the same direction of the force and in the opposite direction with respect to the configuration of figure $1 \mathrm{a}$ and $1 \mathrm{~b}$.

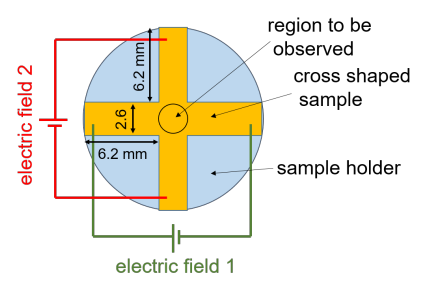

Figure 2: Schematics of the cross shaped sample. The thickness of the cross is about 200 $\mu \mathrm{m}$, the other dimensions are in the figure (in $\mathrm{mm}$ ). The region observed corresponds to the middle of the cross, while the electric connections to apply the perpendicular electric fields are at the arms extremities. Typical values of the applied electric fields are $5 \mathrm{~V} / \mathrm{cm}$. During the application of the electric fields, the samples are heated (Joule effect) to temperatures around $1180 \mathrm{~K}$ to enhance surface diffusion.

We have performed the experiments in a lowenergy-electron microscope (LEEM) to observe in-situ the displacement of 2D nanostructures. To be able to control the motion of a $2 \mathrm{D}$ negative island along a defined path, it is necessary to apply at least two electric fields in two different directions. We have thus developed a specific LEEM sample holder (detailed in the supporting information, see figure S1) to apply two electric fields, labeled E1 and E2, in perpendicular directions. For the observations, we have used a cross-shaped sample (see figure 2), prepared as described in the supporting information. Barreiro et al. ${ }^{26}$ have studied by scanning electron microscopy samples with a geometry similar to that of our crosses. They investigated the evolution of $\mathrm{Al}$ and $\mathrm{Au} 3 \mathrm{D}$ patches that breakdown in smaller objects when electric fields are applied. They showed an effect of the

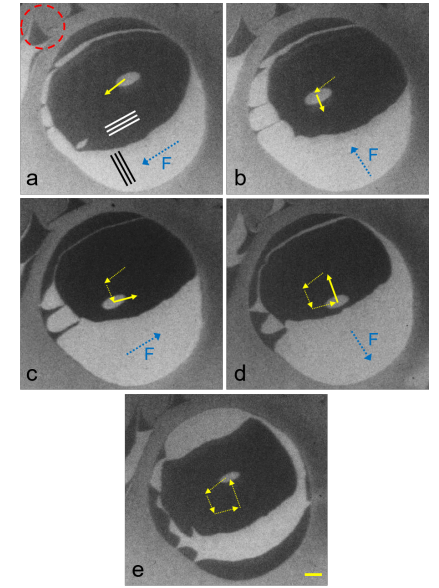

Figure 3: Sequence of dark field LEEM images $(\mathrm{E}=4.5 \mathrm{eV})$ extracted from the movie available in the supporting information. Considering image a taken at time $t_{0}, b, c d$ and e are taken at $\mathrm{t}_{0}+44 \mathrm{~s}, \mathrm{t}_{0}+62 \mathrm{~s}, \mathrm{t}_{0}+131 \mathrm{~s}$ and $\mathrm{t}_{0}+157 \mathrm{~s}$. The yellow scale bar, shown in image e, is $1 \mu \mathrm{m}$. In each image, atomically flat terraces are bright and dark (according to their dimer rows orientation that turns by $90^{\circ}$ on alternated terraces, see the text), while the grey regions correspond to zones with many steps and very shallow terraces. The small white ellipse in the middle is a single-atom-deep 2D hole that moves under the effect of electromigration. The dark terrace surrounding the small bright hole moves opposite to it, because of the mechanisms explained in figure 1 . The regions with multiple steps are less affected by electromigration than flat terraces, and are thus considered reference points. The region in the dashed red circle of figure a does not move and motion of the $2 \mathrm{D}$ hole can be evaluated from that reference point. The direction of the force $\mathrm{F}$ induced by the applied electric field is shown with blue dotted arrows in each image. 
electric-field direction on the object displacement but without control. Details on the conditions of formation of the $2 \mathrm{D}$ negative islands by $\mathrm{Si}$ sublimation, the surface preparation and the LEEM are given in the supporting information. By applying successively E1, E2, -E1 and -E2 we have moved a 2D negative island along a closed loop, as shown by the movie available in the supporting information and summarized in figure 3. The large zone in the center has dark and bright areas, corresponding to atomically flat regions with dimer rows oriented along the [110] and the [1-10] directions alternatively in successive terraces (the dimer rows are schematically represented in figure $3 \mathrm{a}$ with three parallel white or black lines). The small bright ellipse in the center is a 2D one-atom-deep hole inside the elliptical dark terrace that is in turn one-atom lower than the bright larger region around it. The grey regions correspond to areas with many steps and very narrow terraces. Applying E1 $(5 \mathrm{~V} / \mathrm{cm})$ the hole moves from the position shown in figure $3 \mathrm{a}$ in the yellow arrow direction to the position of figure $3 \mathrm{~b}$. Switching off E1 and applying E2, the 2D hole moves to the position shown in figure 3c. The hole is then moved by applying -E1 (with E2=0 V), to the position of figure $3 \mathrm{~d}$. The loop is closed and the hole is moved back to the starting position (figure 3e) by applying -E2 (and E1=0 V). We have thus shown that the motion of a nanoobject can be controlled by electromigration.

Carefully observing the movie and figure 3 it is possible to notice two minor effects that deserve some discussion: the $2 \mathrm{D}$ hole does not exactly follow the expected square loop but rather a trapezoid loop, and the 2D hole area changes with time. The deviation of the hole trajectory from the square loop is due to (i) electric-field and (ii) temperature inhomogeneities.

(i) Because of the section change in the middle of the cross, the orientation of the electricfield lines changes as shown by the simulation of supporting information (figure S2). Therefore, locally, according to the observed area, the direction of the force applied by the electric field could slightly differ from the orientation of the cross arms where the electric field is applied.

(ii) The experiments are performed at high temperatures $(1180 \pm 40 \mathrm{~K})$ to enhance adatom diffusion. At lower temperatures the holes can move but their velocity decreases exponentially with the temperature ${ }^{20}$, while at higher temperatures silicon evaporation increases. The sample is warmer in the regions where the Joule effect is the most effective, corresponding to the cross arms where the electric field is applied, rather than in the cross arms where the electric field is nil. The center of the sample is at intermediate temperatures, because the cold cross arms behave as a heat sink. Furthermore, regions of the sample with different cross sections have different current density and, because of the Joule effect, they heat differently. The temperature differences could lead, through the thermoelectric effect, to secondary electric fields perpendicular to the applied electric field and thus could perturb it. Considering the Si Seebeck coefficient ${ }^{27}$ (about $400 \mu V / K)$, an exaggeratedly-high temperature gradient of $500 \mathrm{~K} / \mathrm{cm}$ (we rather estimate a local gradient of $50 \mathrm{~K} / \mathrm{cm}$ in the sample center) would lead to an electric field of $0.2 \mathrm{~V} / \mathrm{cm}$ that is small with respect to the applied field $(5 \mathrm{~V} / \mathrm{cm})$. Temperature gradients could also lead to the displacement of atoms by thermomigration. However, inverting the electric field in our samples, the temperature gradients do not change but the motion of $2 \mathrm{D}$ negative islands is inverted. Therefore, temperature gradients play only a minor role on the nanostructure migration as compared to the effect of the applied electric field. To evaluate the thermomigration effect we have studied the displacement of $2 \mathrm{D}$ holes in $\mathrm{Si}(001)$ without applying electric fields, in a sample holder specifically developed to apply only a thermal gradient. Preliminary results ${ }^{28}$ show that also in this case $2 \mathrm{D}$ holes move, but their velocity is about one order of magnitude lower than when an electric field is applied.

The size of the 2D holes that we studied changes during the motion, and is affected by two opposite phenomena. The Gibbs-Thomson effect makes $2 \mathrm{D}$ holes shrink. This phenomenon is more important for small holes. For $2 \mathrm{D}$ oneatom-thick positive islands, the concentration of adatoms $c$ in equilibrium with a step with 


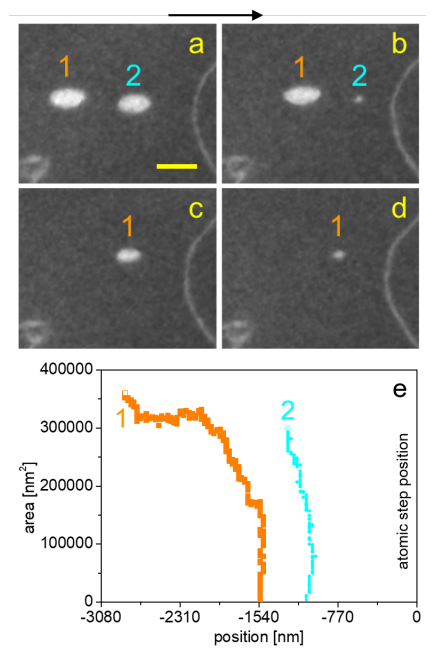

Figure 4: a-d: LEEM images showing the motion of two negative islands from the left to the right, under an electric field oriented to the right. The black arrow represents both the 2Dhole motion and the electric-field direction. The scale bar is is $1 \mu \mathrm{m}$. e: Change of area of the two one-atom-deep holes observed in a-d, as a function of their distance from a step. The position of the step is the reference, at $0 \mathrm{~nm}$, on the right of the graph. The orange squares and the small blue circles show the size of the negative islands on the left and on the right of image a, respectively. The empty symbols show the position and the area of the $2 \mathrm{D}$ holes at the beginning of the experiment. The measured position is that of the 2D-holes center. Hole number 2 shrinks because of adatoms arriving at the left edge (electromigration) while the right edge does not move because it is in a region with very high adatom concentration, due to the vicinity of the step. The error on the size measurements for small areas gives the illusion of a hole going back. radius of curvature $R$ and step edge stiffness $\tilde{\beta}$ is $c \propto \exp \left(\frac{\tilde{\beta}}{R}\right)$ (see for instance ${ }^{29}$ ). A negative island is considered to have negative $R$ in the above formula, and thus the adatom concentration in equilibrium with the hole edges decreases when the hole size decreases. The equilibrium adatom concentration on the surrounding surface is given by the numerous straight steps $(R=\infty)$, and is thus higher than the concentration that would allow $2 \mathrm{D}$ holes to be stable. Therefore adatoms attach to hole edges and 2D holes shrinks. However at our experimental temperatures $(1180 \mathrm{~K})$, evaporation of Si atoms is also active and leads to hole opening. Because of the combination of the two opposite effects, below a critical size, that we estimate from the size-change measurements of several holes in different experiments to be 0.7 $\mu \mathrm{m}^{2}$ at $1180 \mathrm{~K}$, the holes shrink, while above it, they open. Local environment effects, like the distance of a specific 2D hole from other 2D holes/islands, steps, step bunches, impurities or generally defects, also affect the hole size change. Precisely positioning nanostructures at defined distances from defects could be a way to obtain information on local environment effects. For instance, the size of a 2D hole close to a step is expected to decrease fast because of the availability of adatoms coming from the step.

Figure 4a shows two holes (bright ellipses in the LEEM images) obtained by evaporation. One of them (hole 2) has nucleated close to a step (curved bright line on the right of the image). Figures $4 \mathrm{~b}-\mathrm{d}$ show the size change of the two negative islands approaching the step under electromigration. The measured area change is shown in figure 4e. The $2 \mathrm{D}$ hole on the left of figure 4a (orange squares in figure 4e) initially moves keeping a roughly constant area. Then, starting from a distance of $2 \mu \mathrm{m}$ from the step, it finds higher adatom concentration on the terrace and thus atoms leaving from its right edge are more or less compensated by adatoms coming from the nearby step edge and the hole shrinks. The negative island on the right of figure $4 \mathrm{a}$, is generated too close to the step, where the adatom concentration is very high. Therefore, the right edge of the hole does not move 
but atoms continue to arrive on the left edge (similar to the mechanism shown in figure $1 \mathrm{c}-\mathrm{d}$ but without the removal of the black atoms on the right edge of the hole). Thus hole 2 in figure 4 moves only slightly and shrinks, as shown by the small blue circles in figure 4e, because it is too close to the atomic step. Other processes could affect the 2D-hole size changes, like the Schwoebel barrier, anisotropic attachment to (or detachment from) dimer rows. A modification of the scattering properties of electrons due to local electric-field changes close to defects $^{30}$ could also play a role in the size change of 2D holes. The small size changes of the hole shown in figure 3 can be explained in the light of the previous discussion. The hole in figure $3 \mathrm{a}$ has an area of $0.75 \mu \mathrm{m}^{2}$, larger than the critical size, therefore it grows (to $0.86 \mu \mathrm{m}^{2}$ ) during the motion from figure $3 \mathrm{a}$ to $3 \mathrm{~b}$, where it is far from the large-terrace edges. From figure $3 \mathrm{~b}$ to $3 \mathrm{c}$ and $3 \mathrm{~d}$ the hole approaches and runs along an edge, thus its size decreases because of the previously discussed local environment effects. In figure $3 \mathrm{~d}$ the hole area is $0.63 \mu \mathrm{m}^{2}$, below the estimated critical size, therefore it shrinks during the motion towards the position of figure $3 \mathrm{e}$, even if it is far from a terrace edge.

We have made the proof of concept of the control of the motion of nanostructures by means of electric fields. Moving nano-objects close to surface structures or defects can put in evidence local environment effects. In surface science research, as 2D voids feel the presence of steps in their environment and thus change size, they could be used as local probes of surface defects. They should be displaced towards selected regions while monitoring their size. A sudden modification of the size change would give information on the adatom concentration around the surface defect to be probed. We have used as example 2D one-atom-deep holes on a $\mathrm{Si}(001)$ surface, but the method should apply to many systems, as electromigration has been widely observed. For instance, germanium behaves similarly to silicon ${ }^{23}$. 3D particles and 2D surface phases of metals (Au, Ag, $\mathrm{Al}, \mathrm{Ni}, \mathrm{Pd}, \mathrm{In}, \mathrm{Cu}, \mathrm{Pt}$ ) have been observed to move under electric fields ${ }^{31}$. While a direct application of our technique requires to solve some issues (the high temperatures should be reduced and the electric fields increased and applied only locally, the size of the nanostructures should be better controlled), we suggest some perspectives of possible future applications of using two perpendicular electric fields to move nano-objects. Electromigration could become a technique to assemble nanomaterials with a bottom up approach. For instance, we anticipate that the motion of flakes of $2 \mathrm{D}$ materials (like graphene, BN, MoS2, and other transition metal dichalcogenides) under electric fields should be possible, though it has still to be observed. If demonstrated, different flakes could be positioned to assemble nanodevices, for instance to make a transistor. In order to apply the electric fields only locally, micro-scale electrodes should be patterned on a small region of the selected surface. Alternatively, a system of four tips/electrodes could be developed to apply two perpendicular electric fields. It can be envisaged that these tips should be lifted, displaced and applied together at different regions of interest to control locally the motion of nano-objects. A similar setup could be used to locally remove defects from a selected portion of a surface by driving them at the edges of the selected area to obtain atomically flat surfaces. Another possibility to explore is the control of the position with perpendicular electric fields of molecules assembly, macromolecules, DNA chains or biologic units like cells, though a major issue to solve would be the stability of these materials in an electric field.

We thank A. El-Barraj, G. Arthaud, I. Ozerov, M. Dayez and the CINaM-Planete facility for the technical support.

We acknowledge financial support from the Region Sud Provence-Alpes-Côte d'Azur (MELOPEE project).

\section{Supporting Information Available:}

The text file available in the supporting information describes the LEEM imaging mode, the sample holder, the procedure of fabrication of the cross-shaped sample, and the surface preparation. It also shows a simulation of the electric potential in the cross-shaped sample and describes the details of image acquisition used to record the movie included in the supporting 
information. The movie shows the motion of a 2D one-atom-deep hole along a close loop.

This material is available free of charge via the Internet at http://pubs.acs.org.

\section{References}

(1) Huang, J.-A.; Zhao, Y.-Q.; Zhang, X.J.; He, L.-F.; Wong, T.-L.; Chui, Y.-S.; Zhang, W.-J.; Lee, S.-T. Ordered Ag/Si nanowires array: Wide range surfaceenhanced raman spectroscopy for reproducible biomolecule detection. Nano Letters 2013, 13, 5039-5045.

(2) Sciacca, B.; van de Groep, J.; Polman, A.; Garnett, E. C. Solution-grown silver nanowire ordered arrays as transparent electrodes. Advanced Materials 2016, 28, 905-909.

(3) Lynch, M. D.; Patrick, D. L. Organizing carbon nanotubes with liquid crystals. Nano Letters 2002, 2, 1197-1201.

(4) Giermann, A. L.; Thompson, C. V. Solidstate dewetting for ordered arrays of crystallographically oriented metal particles. Applied Physics Letters 2005, 86, 121903.

(5) Trautmann, M.; Cheynis, F.; Leroy, F.; Curiotto, S.; Pierre-Louis, O.; Müller, P. Dewetting of patterned solid films: Towards a predictive modelling approach. Applied Physics Letters 2017, 110, 263105.

(6) Cordoba, R.; Mailly, D.; Rezaev, R.; Smirnova, E.; Schmidt, O. G.; Fomin, V. M.; Zeitler, U.; Guillamon, I.; Suderow, H.; De Tersa, J. M. Three-dimensional superconducting nanohelices grown by $\mathrm{He}+$-focused-ion-beam direct writing. Nano Letters 2019, 16, 1863-1868.

(7) Joachim, C.; Rapenne, G. Molecule Concept Nanocars: Chassis, Wheels, and Motors? ACS Nano 2013, 7, 11-14.
(8) Simpson, G. J.; Garcia-Lopéz, V.; Petermeier, P.; Grill, L.; Tour, J. M. How to build and race a fast nanocar. Nature Nanotechnology 2017, 12, 604-606.

(9) Eigler, D. M.; Schweizer, E. K. Positioning single atoms with a scanning tunneling microscope. Science 1990, 344, 524-526.

(10) Sorbello, R. S. Theory of electromigration. Solid State Physics 1998, 51, 159-231.

(11) Blech, I.; Meieran, E. Electromigration in thin Al films. Journal of Applied Physics 1969, 40, 485 .

(12) Kevrekidis, I. G. External-field-enabled surface nanopattern engineering. Surface Science 2013, 618, 1-2.

(13) Latyshev, A.; Krasilnikov, A.; Aseev, A.; Stenin, S. Effect of electric current on the ratio o the areas of the $(2 \times 1)$ and $(1 \times 2)$ domains at the clean (001) surface of silicon during sublimation. JETP Letters 1989, 48, 526-529.

(14) Latyshev, A.; Litvin, L.; Aseev, A. Peculiarities of step bunching on $\mathrm{Si}(001)$ surface induced by DC heating. Applied Surface Science 1998, 130, 139-145.

(15) Stoyanov, S. Heating current induced conversion between $2 \mathrm{x} 1$ and $1 \mathrm{x} 2$ domains at vicinal (001) Si surfaces - can it be explained by electromigration of $\mathrm{Si}$ atoms? Japanese Journal of Applied Physics 1990, 29, 659-662.

(16) Stoyanov, S. Electromigration induced step bunching on Si surfaces - how does it depend on the temperature and heating current direction. Japanese Journal of Applied Physics 1991, 30, 1-6.

(17) Ichikawa, M.; Doi, T. Study of Si(001) 2x1 domain conversion during direct current and radiative heatings. Applied Physics Letters 1992, 60, 1082.

(18) Métois, J.-J.; Heyraud, J. C.; Pimpinelli, A. Steady-state motion 
of silicon islands driven by a DC current. Surface Science 1999, 420, 250-258.

(19) Doi, T.; Ichikawa, M.; Hosoki, S.; Ninomiya, K. Anisotropic diffusion between the step-up and the step-down directions on a $\mathrm{Si}(001)$ surface. Physical Review $B$ 1996, 53, 16609-16614.

(20) Curiotto, S.; Müller, P.; El-Barraj, A.; Cheynis, F.; Pierre-Louis, O.; Leroy, F. 2D nanostructure motion on anisotropic surfaces controlled by electromigration. $A p$ plied Surface Science 2019, 469, 463-470.

(21) Zandvliet, H. J. W.; Poelsema, B.; Elswijk, H. B. Fluctuations of monoatomic steps on $\mathrm{Si}(001)$. Physical Review B 1995, $51,5465-5468$.

(22) Mo, Y.; Lagally, M. Anisotropy in surface migration of $\mathrm{Si}$ and Ge on $\mathrm{Si}(001)$. Surface Science 1991, 248, 313-320.

(23) Yasunaga, H.; Natori, A. Electromigration on semiconductor surfaces. Surface Science Reports 1992, 15, 205-280.

(24) Ehrlich, G.; Hudda, F. G. Atomic view of surface self diffusion: tungsten on tungsten. The Journal of Chemical Physics 1966, 44, 1039-1049.

(25) Schwoebel, R. Step motion on crystal surfaces. II. Journal of Applied Physics 1969, 40, 614-618.

(26) Barreiro, A.; Rurali, R.; Hernandéz, E.; Bachtold, A. Structured Graphene Devices for Mass Transport. Small 2011, 7, $775-780$.

(27) Fulkerson, W.; Moore, J. P.; Williams, R. K.; Graves, R. S.; McElroy, D. L. Thermal conductivity, electrical resistivity, and Seebeck coefficient of silicon from 100 to 1300 K. Physical Review 1968, 167, 765-782.

(28) El-Barraj, A. Growth and ElectroThermomigration on semiconductor surfaces by Low Energy Electron $\mathrm{Mi}$ croscopy. Ph.D. thesis, Aix Marseille Université, 2019.

(29) Schultze Icking-Konert, G.; Giesen, M.; Ibach, H. Decay of $\mathrm{Cu}$ adatom islands on $\mathrm{Cu}(111)$. Surface Science 1998, 398, 3748.

(30) Tao, C.; Cullen, W. G.; Williams, E. D. Visualizing the electron scattering force in nanostructures. Science 2010, 328, 736740 .

(31) Ichinokawa, T.; Izumi, H.; Haginoya, C.; Itoh, H. Electromigration of metallic islands on the $\mathrm{Si}(001)$ surface. Physical Review $B$ 1993, 47, 9654-9657. 


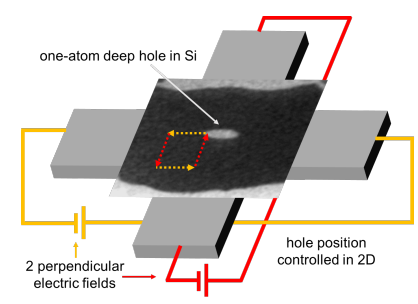

For Table of Contents Only 